\title{
Automatic Segmentation of Spleen based on Anatomical Model and Template Matching
}

\author{
C.H. Dong, X.H. Han, Tomoko TATEYAMA \\ Graduate School of Information Science and Engineering \\ Ritsumeikan University \\ Japan
}

\author{
Y.W. Chen* \\ College of Computer Science and Information Technology \\ Central South University of Forestry and Technology \\ Hunan, China \\ *Corresponding author
}

\author{
Amir H. Foruzan \\ Faculty of Engineering \\ Shahed University \\ Iran
}

\begin{abstract}
Probabilistic atlases based on human anatomy structure have been widely used for organ segmentation, which is used as a prior probability in a Bayes framework. The challenge is how to register the probabilistic atlas to the patient volume. Taking these into consideration, we propose a template matching framework based on the probabilistic atlas for spleen segmentation. Firstly, we find a bounding box of the spleen based on human anatomical localization, which is the statistical geometric location of spleens. Then, the probabilistic atlas is used as a template to find the spleen in this bounding box by using template matching technology. We apply our method into 60 datasets including normal and pathological cases. The Dice/Tanimoto volume overlaps are $0.922 / 0.857$, the root-meansquared error (RMSE) is $1.992 \mathrm{~mm}$. The algorithm is robust to segment normal and abnormal spleens, such as the presence of tumors and large morphological changes. Meanwhile, our proposed method was compared with conventional atlas-based methods. Results demonstrate that segmentation accuracy improved using our method.
\end{abstract}

Keywords-template matching; organ bounding box; spleen segmentation; probabilistic atlas

\section{INTRODUCTION}

Accurate segmentation of abdominal organs is a key step in computer-aided diagnosis (CAD) system. A variety of methods for organ segmentation have been proposed in the literatures. It can be summarized as: classification-based[1-2], region-based[3], contour-based[4], graph cut-based[5]. These segmentation methods are only based on gradient or intensity analysis and do not use any anatomical information. Their performance is not good enough when the image contains noise and the contrast between objects and background is low. Recently, significant effort has been forwarded to the development of anatomical models-based method for organ segmentation, such as probabilistic atlas[6-8], statistical shape model. In anatomical model based methods, the anatomical model can be used as a prior location and shape information of organs. These methods are robust against noise, however, they are sensitive to initialization of their parameters such as initial contour. Our research is focused on the probabilistic atlas- based segmentation method. Currently, rich literatures have been published on probabilistic atlases at the abdominal organs. Probabilistic atlas-based organ segmentation, however, poses a number of challenges. Accurate mapping of the probabilistic atlas onto the input CT volumes is difficult because of the variability in organ shape. Park et al. [6] used an atlas registered with thin plate spline to propagate the segmentation of liver, kidneys, and spinal cord. Using a similar principle, a priori data from probabilistic atlases were used to initialize the segmentation of abdominal organs in [7] and [8]. The construction of these abdominal atlases required user interaction through manual landmarks.

However, there are two limitations of these segmentation methods: (1) Errors of landmark extraction may cause incorrect estimation of the transformation; (2) Even though we can estimate the transformation of landmarks accurately, it is difficult to estimate the accurate transformation of the organ because of the large variation of anatomical structure. Since the bone of the body is considered as a rigid part, we perform registration using the bones in our method. Due to variations in bone shape/size, only depending on one style bone is not enough. Hence, we prepare four different bone references for our segmentation method.

In this paper, we propose a template matching framework based on probabilistic atlas for spleen segmentation. In our proposed method, we first find a bounding box of the organ based on human anatomic structure, which is the relatively rigid characteristics of spleen position; and then the probabilistic atlas is used as a template to find the spleen in this bounding box by the use of template matching.

\section{METHOD}

The proposed method segments the spleen based on a set of prior information. Prior information includes the approximate location of an organ, histogram of the organ's intensities, and a probabilistic atlas of the organ. The organ bounding box is employed to estimate the approximate location of an organ for the observed data. The histogram of the organ's intensity is used to build the likelihood probability 
model (likelihood map). Then, the likelihood map together with the probabilistic atlas is employed in template matching technique. The template matching is proposed to estimate an initial segmentation of the organ. Furthermore, the 'Geodesic Active Contour' (GAC) algorithm [4] guarantees the smoothness of the final result. The framework of our proposed method is shown in Fig. 1.

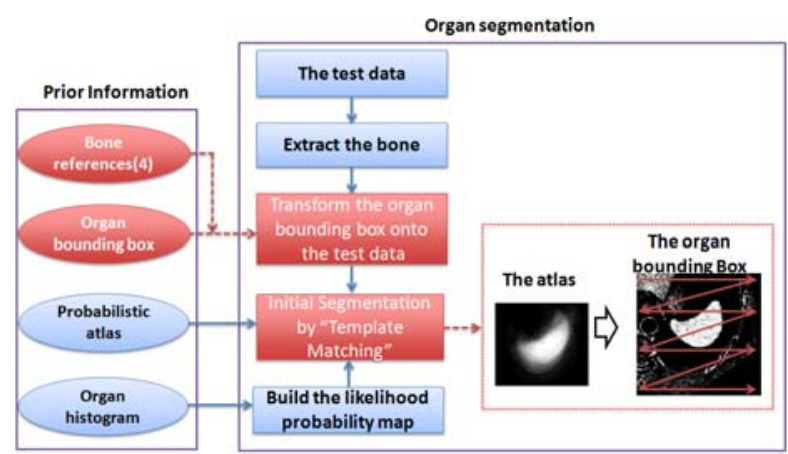

FIGURE I. THE FRAMEWORK OF OUR PROPOSED SEGMENTATION METHOD.

\section{A. Organ Bounding Box Construction}

The development of bounding box-based method is a new trend for organ segmentation, which can be easily extracted from CT volumes. Our algorithm incorporates atlas concept within a bounding box construction by warping the bone.

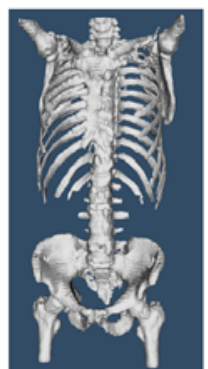

(a)

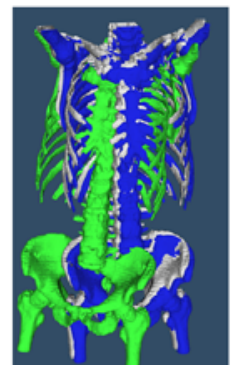

(b)

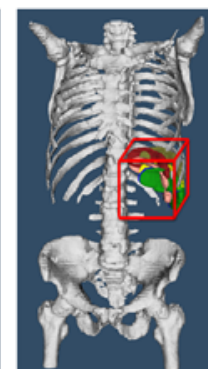

(c)
FIGURE II. AN EXAMPLE OF THE SPLEEN BOUNDING BOX

CONSTRUCTION. (A) EXAMPLES OF BONE EXTRACTION; (B) REGISTRATION OF BONES; (C) THE ENSEMBLE OF SIX SPLEENS WITH ITS BOUNDING BOX.

For bone extraction, the first step of our method is finding the abdominal region using the Otsu thresholding. Next, we model the intensity range of bones by a 'Dynamic Gaussian Mixture'. Then, we employ region-growing algorithm to segment bone, and use morphological operators to refine the results. As explained above, due to the variation of bone, we prepare four different bone references. We use an affine transform for the registration.

To build a bounding box of spleen, we firstly extracted the bone of the input data. Then, using the adaptive selection of appropriate reference bone, the extracted bone from the input data is registered to the reference by an affine transform. Thus, the segmented spleen mask of this input data can be transformed by the same transformation. Finally, after registration for all the training data, we find the volume of interest of the ensemble of spleens and use it as the bounding box of organ. Fig. 2 give an example of the spleen bounding box based on six cases.

\section{B. Likelihood Probability Model}

Likelihood probability model (or likelihood map) of an organ refers to an image in which the intensity of a pixel corresponds to the probability of belonging to that organ. To reduce the irrelevant neighbouring tissues from the input candidate organ region, the Gaussian model is applied for analyzing the input organ intensity distribution by the following equation.

$$
P(\text { Intensity } \mid \text { Spleen })=P\left(y_{i} \mid i \in \text { Spleen }\right)=\frac{1}{\sqrt{2 \pi \sigma}} \exp \left(\frac{-\left(y_{i}-\mu\right)^{2}}{2 \sigma^{2}}\right)
$$

The Gaussian parameters mean $\mu$ and variance $\sigma^{2}$ could be estimated by observing the density histogram of the spleen. A likelihood map for spleen is generated that the intensities of the pixels in the original CT image are converted into a probability using the histogram of the spleen, as shown in Fig. 3.

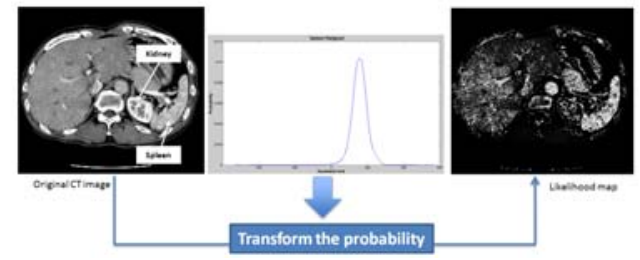

FIGURE III. ESTIMATION OF THE SPLEEN PROBABILITY MAP ON CT IMAGE.

\section{Probabilistic Atlas Model}

The organ probabilistic atlas can serve as a prior probability map of the spleen. The segmented spleen masks in the training data were used to build a probabilistic atlas for the spleen. A single training spleen mask is arbitrarily chosen as the reference image, and the rest of the spleen masks are regarded as the moving images and registered to this reference. In our proposed segmentation method, the probabilistic atlas is used as a template to detect the organ in the organ bounding box by the use of template matching technology. Thus, when the probabilistic atlas is constructed, we select the largest region that containing all the input organ masks as our atlas template.

\section{Template Matching}

Template matching is one of the key innovations of our algorithm. The template matching is based on the idea that those pixels, which belong to organ, have similar intensities to the organ's histogram and their locations are in parts of the image where organ may be found with a higher probability. We use prior information of the spleen bounding box to restrict the processing region in template matching. The template matching algorithm moves the probability atlas in the bounding box of the spleen and multiplies the probability atlas by the likelihood map. The summation of probabilities of pixels of the result is used as an index $\mathbf{j}=\left[j_{x}, j_{y}, j_{z}\right]$. The output of the template matching which corresponds to the maximum index is used to find the best location of the initial segmentation of the spleen. 


$$
\begin{gathered}
P(\text { Spleen } \mid \text { Intensity })=\underset{\mathbf{j}_{\max }}{\arg \max }\left[\sum_{\mathbf{j}} P(\text { Intensity } \mid \text { Spleen }) \times T_{\mathbf{j}}(P(\text { Spleen }))\right] \\
\text { where } \quad j_{x} \in\left[j_{\text {xstart }}, j_{\text {xend }}\right], \quad j_{y} \in\left[j_{\text {ystart }}, j_{\text {yend }}\right]
\end{gathered}
$$
and $j_{z} \in\left[j_{\text {zstart }}, j_{\text {zend }}\right]$. The probabilistic atlas can be regarded as the template to search the best pose from the beginning of the region $\left(j_{x s t a r t}, j_{y s t a r t}, j_{z s t a r t}\right)$ to the end of the region $\left(j_{\text {xend }}, j_{\text {yend }}, j_{\text {zend }}\right)$. Moreover, $T(\cdot)$ represents the translation of the spleen probabilistic atlas.

\section{RESULTS}

Our dataset comprised $60 \mathrm{CT}$ volumes, including 55 data with a resolution of $0.683 \times 0.683 \times 1 \mathrm{~mm} 3$ and a size of $512 \times$ $512 \times(185-263)$ and the other 5 data with a resolution of 0.663 $\times 0.663 \times 1.2 \mathrm{~mm} 3$ and a size of $512 \times 512 \times(309-607)$. The spleen masks were manually segmented in each data by the experienced experts. For our dataset, 38 data were randomly chosen as the training data for constructing the organ bounding box and probabilistic atlas, the remaining 22 were used for testing the performance.

The dice coefficient (Dice) and Tanimoto volume overlap (VO) are the most popular methods to evaluate segmentation accuracy. These two metric are given in percent and based on the voxels of two binary 3D volumes, $V_{\text {manual }}$ as the manually and $V_{\text {auto }}$ as the automatically segmented organ.

$$
\begin{aligned}
& \text { Dice }=\frac{2\left|V_{\text {manual }} \cap V_{\text {auto }}\right|}{\left|V_{\text {manual }}\right|+\left|V_{\text {auto }}\right|} \times 100 \% \\
& V O=\frac{\left|V_{\text {manual }} \cap V_{\text {auto }}\right|}{\left|V_{\text {manual }} \cup V_{\text {auto }}\right|} \times 100 \%
\end{aligned}
$$

In addition, we use the root mean square error (RMSE) to estimate the accuracy based on the surface points of two 3D volumes.

In order to make a comparison, we showed the result using conventional method (Con_Seg), in which the transformation between the reference bone and the patient bone was directly applied to the atlas, in Fig.4(c). The white regions had a higher probability of belonging to spleen. Comparison the original CT image (Fig.4(a)) with the corresponding likelihood map (Fig.4(b)), it revealed that the spleen can be more easily distinguished from other tissues in the likelihood map. However, if we combined the atlas with likelihood map, the other tissues with similar intensity would be significantly reduced, as shown in Fig. 4(c) and (d). Moreover, it can also be seen that there were still a lot of other tissues in the conventional method even we used a probabilistic atlas, because we cannot align the atlas to the patient volume accurately, while our proposed method can achieve more accurate alignment. Template matching was utilized to improve the accuracy of the registration.

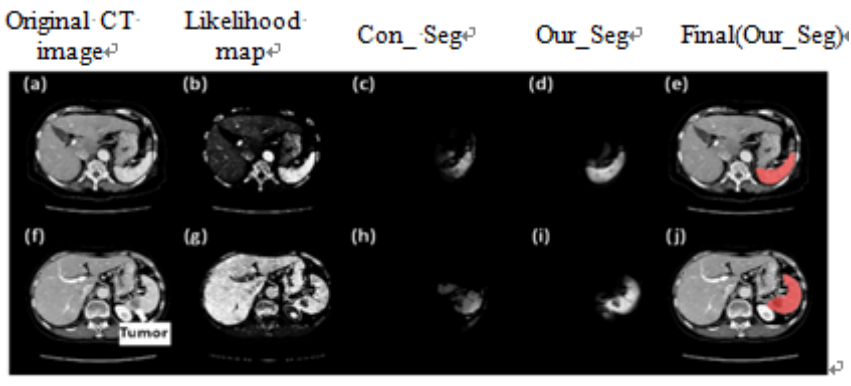

FIGURE IV. COMPARISON OF SEGMENTATION RESULTS BY CONVENTIONAL METHOD AND OUR PROPOSED METHOD WITH TWO DIFFERENT PATIENTS. THE FIRST ROW IS CASE 10 (NORMAL) AND THE SECOND ROW IS CASE 11 (PATHOLOGICAL)

Additionally, a pathological spleen was segmented in Fig. 4(f)-(j). The results demonstrated the robustness of our proposed algorithm in the presence of tumors. As explained as the above, the template matching was aimed to estimate an initial segmentation of the spleen. Furthermore, the GAC algorithm was applied to obtain the final result, as shown in Fig. 4(e)-(j). The red ones were segmented spleen slice, which are overlaid with the original CT slice. It can be seen that accurate segmentations have been achieved.

TABLE I. ACCURACY OF OUR PROPOSED METHOD WITH DIFFERENT METRICS.

\begin{tabular}{|c|c|c|c|}
\hline Mean & Dice & VO & RMSE \\
\hline Con_Seg & 0.606 & 0.463 & 3.104 \\
\hline Our_Seg & 0.922 & 0.857 & 1.992 \\
\hline
\end{tabular}

Table 1 listed the average of Dice, VO and RMSE between automated and manually segmentations for all 22 test CT scans. It indicated that there was a significant difference in all the metrics when compared results using Con_Seg with those using Our_Seg. The simulation has verified that the performance of Our_Seg was much better than Con_Seg for segmenting the spleen.

\section{CONCLUSION}

In this paper, we proposed a template matching framework based on probabilistic atlas for spleen segmentation. In our proposed method, we first found a bounding box of the spleen, and then the probabilistic atlas was used as a template to find the spleen in the bounding box using template matching technology. Compared with the conventional method, our proposed method can significantly improve both segmentation accuracy and robustness. As the works for the future, the proposed method can be extended to segment other organs.

\section{ACKNOWLEDGEMENT}

This work is supported in part by the Grant-in Aid for Scientific Research from the Japanese Ministry for Education, Science, Culture and Sports (MEXT) under the Grant No. 2430076, No. 24103710, in part by the MEXT Support Program for the Strategic Research Foundation at Private Universities (2013-2017), and in part by the R-GIRO Research Fund from Ritsumeikan University. 


\section{REFERENCES}

[1] Kasiri K., Kazemi1 K., Dehghani M.J., Helfroush M.S., Atlas-based Segmentation of Brain MR Images Using Least Square Support Vector Machines, IEEE Conference on Image Processing Theory Tools and Applications, IPTA 2010, pp. 306-310, 2010.

[2] van Rikxoort Y. A.E., van Ginneken B., Automatic segmentation of the liver in computed tomography scans with voxel classification and atlas matching, MICCAI Workshop 3-D Segmental. Clinic: A Grand Challenge, pp. 101-108, 2007.

[3] Adams R., Bischof L., Seeded Region Growing, IEEE Transactions on Pattern Analysis and Machine Intelligence, 16(6), pp. 641-647, 1994.

[4] Foruzan A.H., Chen Y. W., Zoroofi R.A., Furukawa A., Sato Y., Hori M., Momiyama N., Segmentation of Liver in Low-contrast Images Using K-Means Clustering and Geodesic Active Contour Algorithms, Journal of IEICE Transactions on Information and Systems, E96-D, pp.798-807, 2013.

[5] Boykov Y., Graph Cuts and Efficient N-D Image Segmentation, International Journal of Computer Vision, vol.70, no.2, pp.109-131, 2006.

[6] Park H., Bland P.H., Meyer C.R., Construction of an abdominal probabilistic atlas and its application in segmentation, IEEE Transactions on Medical Imaging, 22, pp. 483-492, 2003.

[7] Zhou X., Kitagawa T., Hara T., Fujita H., Zhang X., Yokoyama R. Kondo H., Kanematsu M., Hoshi H., Constructing a Probabilistic Model for Automated Liver Region Segmentation Using Non-contrast X-Ray Torso CT images, IEEE International Conference on Medical Image Computing and Computer-Assisted Intervention, MICCAI 2006, pp. 856-863, 2006.

[8] Linguraru M.G., Sandberg J.K., Li Z., Shah F., Summers R.M., Automated segmentation and quantification of liver and spleen from CT images using normalized probabilistic atlases and enhancement estimation, International Journal of Medical Physics, 37(2), pp. 771-783, 2010 